\title{
Fast and Robust Fingerprint Identification Algorithm and Its Application to Residential Access Controller
}

\author{
Hiroshi Nakajima ${ }^{1}$, Koji Kobayashi ${ }^{2}$, Makoto Morikawa ${ }^{3}$, Atsushi Katsumata ${ }^{3}$, \\ Koichi Ito ${ }^{4}$, Takafumi Aoki ${ }^{4}$, and Tatsuo Higuchi ${ }^{5}$ \\ ${ }^{1}$ Building Systems Company, Yamatake Corporation, \\ 54 Suzukawa, Isehara, Kanagawa 259-1195 Japan \\ ${ }^{2}$ Building Systems Company, Yamatake Corporation, \\ 2-15-1 Kounan, Minato, Tokyo 108-6030 Japan \\ ${ }^{3}$ Research and Development Center, Yamatake Corporation, \\ 1-12-2 Kawana, Fujisawa, Kanagawa 251-8522, Japan \\ ${ }^{4}$ Graduate School of Information Science, Tohoku University, \\ 6-6 Aoba, Aramaki, Aoba, Sendai, Miyagi 980-8579, Japan \\ ${ }^{5}$ Faculty of Engineering, Tohoku Institute of Technology, \\ 35-1 Kasumi, Yagiyama, Taihaku, Sendai, Miyagi 982-8577, Japan
}

\begin{abstract}
A novel fingerprint recognition algorithm suitable for poor quality fingerprint is proposed, and implementation considerations to realize fingerprint recognition access controllers for residential applications are discussed. It is shown that optimizing spatial sampling interval of fingerprint image has equivalent effect of optimizing high limit frequency of low-pass filter in the process of phase based correlation. The processing time is $83 \%$ shorter for the former than the latter. An ASIC has been designed, and it is shown that fingerprint matching based access controller for residential applications can be successfully realized.
\end{abstract}

\section{Introduction}

Biometrics has been recognized as indispensable means to attain security in various areas of social life. Fingerprint is the most frequently used, because it exhibits higher performance by smaller size at lower cost than other biometrics [1,2,3].

It is widely recognized that there are some percentage of people whose fingerprint is difficult for automatic recognition. Typical cases include senior citizens whose finger skin tend to be flat, house wives who uses fingertip hard, or those who suffer skin diseases such as atopic dermatitis. In general, pressure sensitive fingerprint sensor [4] produces better images than optical sensors or various types of semiconductor fingerprint sensors in cases when fingertip is dry or wet. However, when the problem stems from structure of finger surface itself, some other approaches have to be taken.

The authors have been studying a pattern-matching algorithm named Phase-Only Correlation [5]. POC is not only good for biometrics such as fingerprint, but also for sub-pixel precision translation measurements for industrial applications [6]. BandLimited POC (BLPOC) is modified POC in that high frequency components are eliminated in the process of POC calculations [7].

Typical fingerprint recognition algorithm extracts lineal structure from the image. Such kinds of methods are referenced as minutiae algorithms in this paper. The struc- 
tural reproducibility is especially important for minutiae algorithms in order to reduce false rejections for genuine attempts. It has been shown that BLPOC improves fingerprint recognition performance especially when numbers of images from those who have poor quality fingerprints are included.

On the other hand, POC based algorithms require more computational resources than minutiae algorithms in general, because the algorithms are based on twodimensional discrete Fourier transformation (DFT). It is too much burden for typical microprocessors to process a fingerprint image in a moment. However, the algorithm is suited for hardware implementation such as ASIC, because DFT is calculated by repetitive executions of sum-of-products arithmetic.

In this paper, a novel fingerprint recognition algorithm that has as good recognition performance as BLPOC is described. The effect of eliminating high frequency components of BLPOC is now realized by optimizing spatial sampling interval of fingerprint image. The computational time for the proposed algorithm is $83 \%$ shorter than that for BLPOC. The recognition performance is evaluated using fingerprint database in comparison with BLPOC and a typical minutiae algorithm.

The CPU burden for the algorithm is still high, and therefore an ASIC has been implemented. The architecture of the ASIC is based on pipelining. Required functions such as re-sampling and scaling are executed in pipeline fashion with DFT calculation, therefore, the time for those functions can be eventually neglected. The processing time is 110 times faster for the ASIC than a typical personal computer. As a result, a prototype of compact access controller for residential applications that uses the algorithm, the ASIC, and a pressure sensitive fingerprint sensor can be realized.

\section{Phase-Based Fingerprint Recognition Algorithm}

\subsection{Proposed Fingerprint Recognition Algorithm}

The fingerprint recognition algorithm using BLPOC is described as following steps of processes. Refer [7] for more details of definitions of POC and BLPOC.

(a) Rotation Alignment

Let $f$ be an input fingerprint image and $g$ be a registered image. For each image $f_{\theta}$ rotated by $\theta$ in 1 degree step, $-20^{\circ} \leq \theta \leq 20^{\circ}$, of $f$, compute POC function $\hat{r}_{f g}$ with $g$. $\Theta$ is the angle of $f_{\theta}$ that produces the highest peak value of the POC function. $f_{\Theta}$ is defined as the rotationally aligned image of $f$.

(b) Translation Alignment

$\hat{r}_{f g}$ also gives the amount of two-dimensional translation displacement $\delta$ as the location of the peak. Align $f_{\Theta}$ and $g$ by using $\delta$. Let $f^{\prime}$ and $g^{\prime}$ be the resultant translation aligned images.

(c) Conjuncture Area Extraction

Let $f^{\prime \prime}$ and $g^{\prime \prime}$ be the part of $f^{\prime}$ and $g^{\prime}$ where the fingerprint image is common.

(d) Upper Limit Frequency Calculation

Calculate upper limit frequencies of $\left(K_{1}, K_{2}\right)$ as inherent frequency band by using two-dimensional DFT. 
(e) BLPOC Calculation

Calculate BLPOC function $\hat{r}_{f "}^{K_{1} K_{2}}$ from $f^{\prime \prime}$ and $g^{\prime \prime}$ using $\left(K_{1}, K_{2}\right)$.

(f) Score Calculation

The BLPOC score is defined as sum of two largest peak values of BLPOC function.

The essential part of BLPOC is in step (e) above, where $K_{1}$ and $K_{2}$ are adaptively determined per individual fingerprint image pair. Hardware implementation of BLPOC may not be straightforward because the size of the images varies.

In our experiments using pressure sensitive fingerprint sensor [4], BLP-100 $384 \times 256$ pixels, and $0.058 \times 0.058 \mathrm{~mm}$ pixel pitch, the optimum values of $K_{1}$ and $K_{2}$ ranges roughly 0.4 to 0.6 . It is expected that selecting value of 0.5 may not produce significant performance differences.

Widening spatial re-sampling interval of an original image has similar effects as of lowering cutoff frequency of low-pass filter. It is assumed that the effect of aliasing stemmed from re-sampling can be neglected. Setting high limit cut-off frequencies of BLPOC is replaced by wider spatial re-sampling interval. Indices for DFT and inverse DFT are selected to be constants. Conjuncture area extraction and improving score calculation function are simplified as well.

The processes of the proposed algorithm significantly simplify aforementioned BLPOC processes as follows.

(a)

\section{Re-sampling}

Images $f$ and $g$ are re-sampled by scaling factor of $S$. The resultant image is defined as constant size of $128 \times 128$ pixels, because DFT calculation is faster for 2's power indices than arbitrary indices. The center of re-sampled image is moved to the gravity center of the original image instead of adjusting translation deviation. This is considered to be simplified version of BLPOC steps (b) and (c).

(b) Rotational Alignment

For each images $f_{\theta}$ rotated by $\theta$ in one degree step, $-20^{\circ} \leq \theta \leq 20^{\circ}$, of $f$, compute POC function $\hat{r}_{f_{\theta} g}$ with $g$. This process corresponds to step (a) of BLPOC.

(c) Score Calculation

The three largest peaks within $5 \times 5$ pixels from the maximum peak are evaluated. The evaluation function to get score value is either the value of the maximum peak, or the sum of peak values weighed by the inverse of the distance from the maximum peak. The distance has offset value of 1 , therefore weight is 1 for the maximum peak. The reason of the weight function is that POC function of imposter calculations tends to produce large peaks in far location from the maximum peak.

\subsection{Performance Evaluations}

The ratio of those who have difficult fingerprint pattern is intentionally increased to create fingerprint database for performance evaluation. Total of 12 subjects, 8 males and 4 females, are participated. Seven of them have fine fingerprint condition, three dry finger, one rough finger skin, and one atopic dermatitis skin lesion. The typical ratio of difficult fingerprint person of some percent is intentionally higher here, $41.6 \%$, for this database. 
Ten fingerprint images are taken from each subject. The genuine match combinations are ${ }_{10} C_{2} \times 12=540$, and imposter combinations ${ }_{120} C_{2}-540=6600$.

The first experiment is to test POC recognition performance by varying spatial sampling interval in order to verify that widening of spatial sampling interval has equivalent effect of lowering cutoff frequency of low-pass filter by BLPOC. The results are shown in Figure 1. The original image from BT-100 is re-sampled by the factor of $100 \%$ to $30 \%$ in $5 \%$ steps. Note that the sampling interval is converted to bits per inch (BPI) by using the sensor's 0.058 micrometer dot pitch. EER and zero FMR values are plotted per sampling interval by two evaluation functions. Zero FMR values may be less significant, because the size of database is small for this evaluation. The first evaluation function simply uses the value of the largest peak. The second one uses aforementioned weighed and averaged peak values. The EER and zero FMR of BLPOC is also shown in the figure as references. 200 DPI sampling produces the best performance, and it is equivalent to that of BLPOC as shown in the figure. The result also implies that the cost of fingerprint sensor can be further reduced by realizing, possibly low-cost, low-resolution sensor.

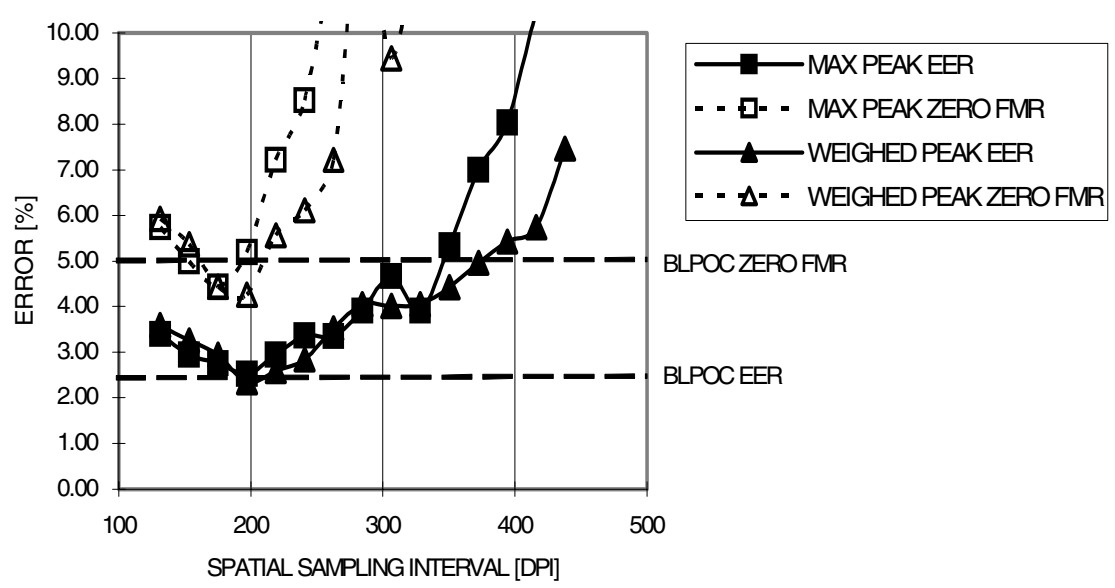

Fig. 1. Characteristics of Spatial Sampling Interval

The second experiment is to compare the performance of the algorithm with that of a minutiae algorithm and BLPOC. The EER and zero FMR values are summarized in Table 1, and ROC characteristics are shown in Figure 2. Again, zero FMR values may be less significant for this small database. The proposed algorithm shows as good performance as that of BLPOC, and both are superior to the minutiae algorithm.

The proposed algorithm can be processed considerably faster than BLPOC. The CPU time to calculate the proposed algorithm using a personal computer of Pentium 4, 3.06GHz, using MATLAB 7.01 is $19.07 \mathrm{~s}$ and $2.45 \mathrm{~s}$ for BLPOC and the proposed algorithm, respectively. 


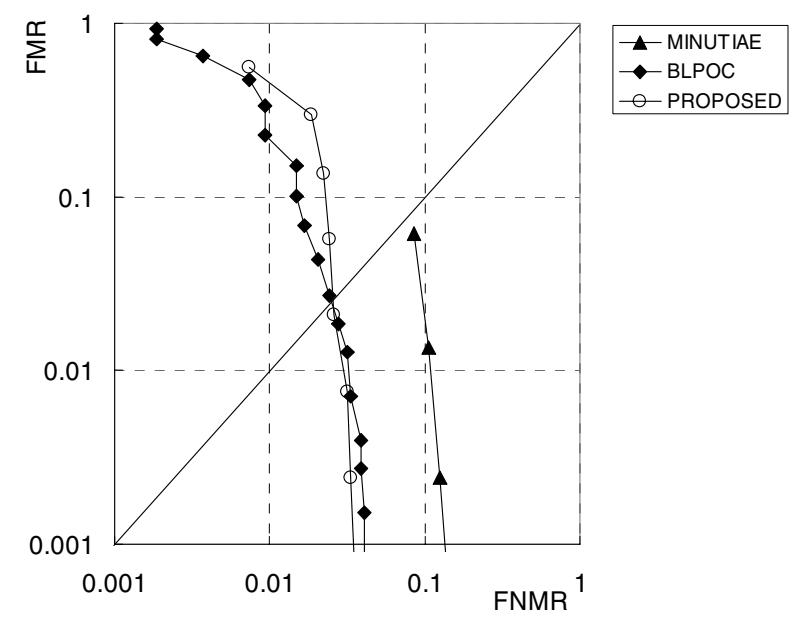

Fig. 2. ROC Comparison

Table 1. Summery of Performance Comparison

\begin{tabular}{|c||c|c|}
\hline & EER [\%] & $\begin{array}{c}\text { ZeroFMR } \\
{[\%]}\end{array}$ \\
\hline \hline MINUTIAE & 7.34 & 17.41 \\
\hline BLPOC & 2.46 & 5.00 \\
\hline PROPOSED & 2.34 & 4.26 \\
\hline
\end{tabular}

\section{LSI Implementation}

There have been POC dedicated LSI implementations reported [8, 9, 10]. ASIC approach is very important for residential applications, because it reduces number of components while processing POC algorithm in a moment.

An ASIC has been developed. The picture is shown in Figure 3, and the block diagram in Figure 4. The pipeline architecture is fully adopted. The fingerprint image signal is re-sampled, and the output image is $128 \times 128$ pixels. The image goes through the internal memory bus, and fed into the local memory through the post-processing controller. The controller calculates the image parameters such as average brightness, and maximum brightness. The image interface, resizing, and image parameter measurements are processed in pipeline fashion with data transfer, and therefore the processing time for those functions can be neglected effectively. Image in the local memory are next read to internal memory through the pre-processing controller, and it eliminates offset and converts real data to complex data for succeeding DFT calculation again in pipeline fashion. The internal memory is divided into four blocks, each of which is for two pairs of horizontal lines. One pair is for input image, and the other pair for registered image. As soon as a line of data transfer is completed and DFT conversion has started, transfer of the next line to the other buffer is started. Therefore, the data transfer time can be neglected. The output data of the DFT unit goes to the local memory 
through post-processing controller, and the data can be scaled by the multiplexer, or converted to phase in order to minimize registration data size for storage. In this way, the ASIC removes most of heavy POC related burdens from CPU.

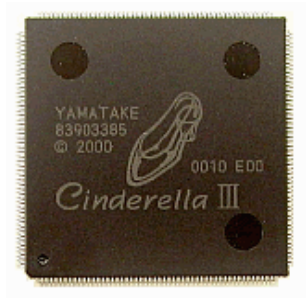

Fig. 3. Picture of the ASIC

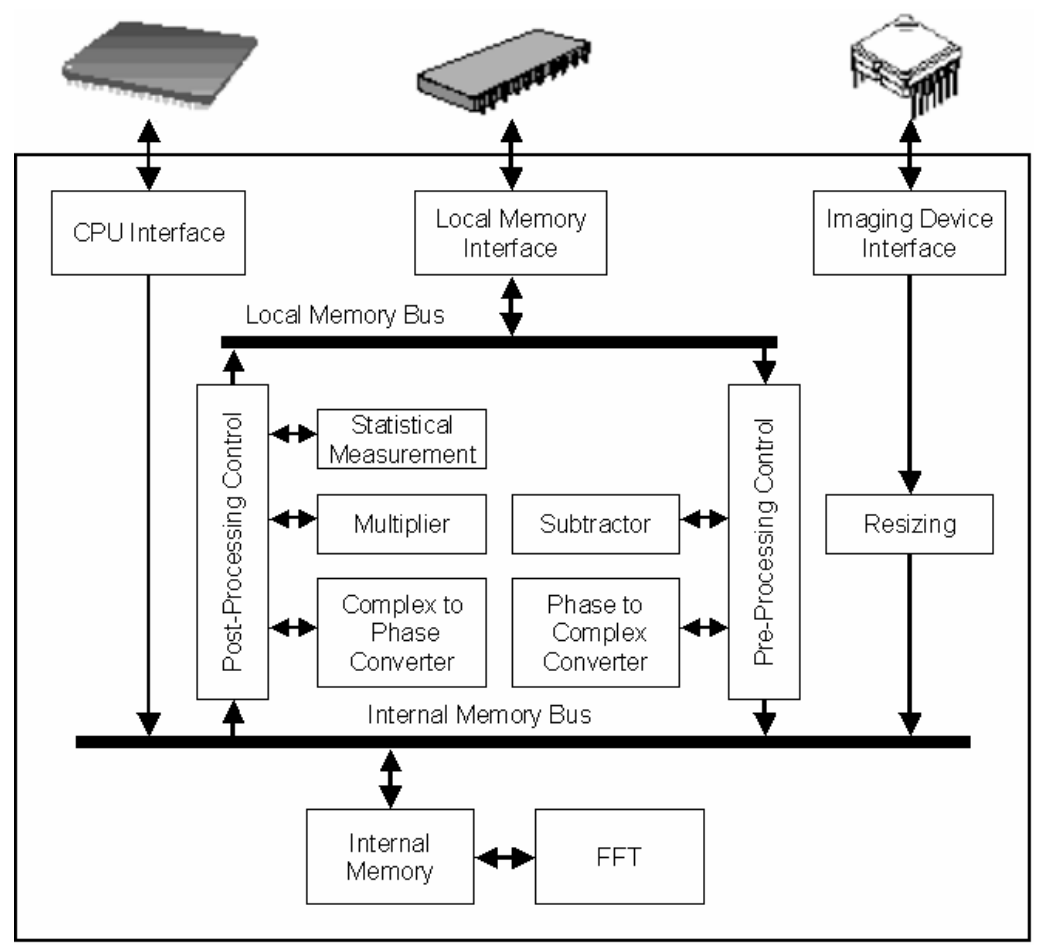

Fig. 4. ASIC Block Diagram

The throughput of the ASIC is compared with that of a typical personal computer. The time for fundamental $128 \times 128$ POC calculation is $8.8 \mathrm{~ms}$ at $57 \mathrm{MHz}$ clock, whereas the same calculation takes average of $28 \mathrm{~ms}$ by aforementioned PC. The performance of the LSI is $\frac{28}{8.8} \times \frac{3060}{57} \cong 171$ times higher than PC, if the performance is compared in a normalized clock frequency. 


\section{Fingerprint Access Controller for Residential Applications}

The most important feature of the fingerprint recognition access controller for residential applications is to realize a good product for ordinary people, especially for senior citizens or housewives who tends to have poor quality fingerprints and frequently at rough conditions. Pressure sensitive fingerprint sensor is applied, because it is insensitive to wet or dry fingers. The ASIC processes a verification calculation at 0.3 second.

The prototype has a graphical LCD display unit, and it provides various userfriendly interface capabilities. Fingerprint image is displayed in case when fingertip is mistakenly placed and the sensor cannot take adequate image. Figure 5 shows the picture of the prototype.

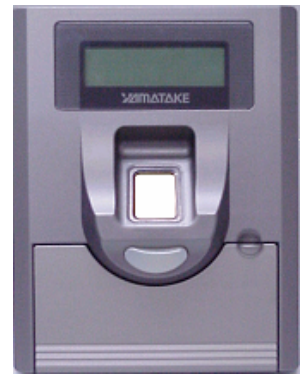

Fig. 5. Fingerprint Access Controller Prototype

\section{Summary}

It has been shown that by optimizing spatial sampling interval of fingerprint image, the POC recognition performance is improved as good as BLPOC while reducing processing time dramatically. An ASIC has been implemented, and a prototype of fingerprint recognition access controller has been realized successfully. Because the algorithm is robust to those who have poor quality fingerprint, and the application products can be simple and cost-effective by using the ASIC, the resultant fingerprint recognition access controller can be ideal for residential applications.

It is noticeable that the POC based algorithms, including the one described in this paper, are less dependent on the structure of target images, and therefore they are good for other biometrics. For examples, POC exhibits excellent recognition performance for iris recognition [11]. It also has been tested as three-dimensional human face measurements $[12,13,14]$. POC also calculates parallax in one-hundredth resolution using a pair of images taken by cameras set in parallel for the case.

\section{References}

1. Wayman, J., Jain, A., Maltoni, D., Maio, D.: Biometric Systems. Springer (2005)

2. Maltoni, D., Maio, D., Jain, A. K., Prabhakar, S.: Handbook of Fingerprint Recognition. Springer (2003) 
3. Jain, A. K., Hong, L., Pankanti, S., Bolle, R.: An Identity Authentication System Uusing Fingerprints. Proc. IEEE, Vol.85, No.9 (1997) 1365-1388

4. http://www.bm-f.com/

5. Nakajima, H., Kobayashi, K., Kawamata, M., Aoki, T., Higuchi, T.: Pattern Collation Apparatus based on Spatial Frequency Characteristics. US Patent 5,915,034 (1995)

6. Takita, K., Aoki, T., Sasaki, Y., Higuchi, T., Kobayashi, K.: High-accuracy Subpixel Image Registration Based on Phase-only Correlation," IEICE Trans. Fundamentals, Vol.E86A, No.8 (2003) 1925-1934

7. Ito, K., Nakajima, H., Kobayashi, K., Aoki, T., Higuchi, T.: A Fingerprint Matching Algorithm Using Phase-only Correlation. IEICE Trans. Fundamentals, Vol.E87-A, No.3 (2004) 682-691

8. Morikawa, M., Katsumata, A., Kobayashi, K.: Pixel-and-Column Pipeline Architecture for FFT-based Image Processor. Proc. IEEE Int. Symp. Circuit and Systems, Vol.3 (2003) 687-690

9. Morikawa, M., Katsumata, A., Kobayashi, K.: An Image Processor Implementing Algorithms using Characteristics of Phase Spectrum of Two-dimensional Fourier Transformation. Proc. IEEE Int. Symp. Industrial Electronics, Vol.3. (1999) 1208-1213

10. Miyamoto, N., Kotani, K., Maruo, K., Ohmi, T.: An Image Recognition Processor using Dynamically Reconfigurable ALU. Technical Report of IEICE, ICD2004-123. (2004) 13 18 (in Japanese).

11. Miyazawa, K., Ito, K., Aoki, T., Kobayashi, K.: A Design of an Iris Matching Algorithm based on Phase-only Correlation. Int. Conf. Image Processing (2005) (in press)

12. Takita, K, Muquit, M. A., Aoki, T, Higuchi, T.: A Sub-Pixel Correspondence Search Technique for Computer Vision Applications. IEICE Trans. Fundamentals, Vol.E87-A, No.8. (2004) 1913-1923

13. http:/www.aoki.ecei.tohoku.ac.jp/poc/

14. Uchida, N., Shibahara, T., Aoki, T., Nakajima, H, Kobayashi, K.: 3D Face Recognition using Passive Stereo Vision. Int. Conf. Image Processing (2005) (in press) 